\title{
PINK PUFFER and BLUE BLOATER PROFILE in ACUTE STROKE
}

Fiori Patrizia, Corbo Antonio, lorillo Luigi, Pelosi Chiara, Savino Patrizia, Bellizzi Gennaro, Dragonetti Carmela, Alberigo Marilisa, De Caro Monica, Guerriero Barbara, Pace Erminio, Raffa Mariangela, Giannetti Luigi Maria, Mazza Emerico, Tammaro Carmine, Monaco Antonio

S. Ottone Frangipane Hospital, ASL AV, Ariano irpino (AV) - University of Naples

\section{Introduction}

Cardiac biomarkers are increased in cerebrovascular conditions, especially in class III/IV, C/D New York Heart Association (NYHA) and American Cardiology Association (ACA) scales. The aim of our current study is to evaluate the impact of respiratory failure and the differences between pink puffers and blue bloaters.

\section{Materials and Methods}

So far, we recruited 431 acute strokes (AS, age 79,73 sd 10,49), 121 chronic cerebrovascular conditions (CCVD, age 79,08 sd 8,81), 25 other neuropsychiatric diseases (OND, age 50,88 sd 16,02). They underwent ECG and chest ray and/or CT at emergency department, blood withdrawal within 24 hours, echocardiography within one week.

\section{Results}

Preliminary results show significant lower $\mathrm{O}_{2}$ saturation and higher levels of $\mathrm{pCO}_{2}$, heart rate, troponin ths (tro ths) and N-terminal-pro-Brain Natriuretic Peptide (NTpro-BNP) levels in class III/IV, C/D NYHA and ACA blue bloater AS (group A) compared to pink puffer AS (group B) (Fig. 1a, 1b, 2a, 2b, 3).
Better outcomes at Glasgow Coma Scale (GCS) and Modified Rankin Scale (MRS) were observed in the latter compared to the former (Fig. $4 a$ and $4 b$ ).

\section{Conclusions}

Respiratory failure worsens ischaemic sufferance and negatively interferes with recovery. High pressure and volume overload in blue bloaters increase the risk of cardiac ischaemic damage with subsequent poor neurological outcomes. Diuretics and novel therapeutical agents, as recombinant brain natriuretic peptide and sacubitril, may be pivotal in restoring circulatory function in these patients. Bridge therapy with low molecular heparins may be useful for preventing thromboembolism. Non-Invasive Positive Pressure Ventilation may be helpful in increasing perfusion and limiting diffusion-restricted volume in both pink puffers and blue bloaters, particularly in the latter.
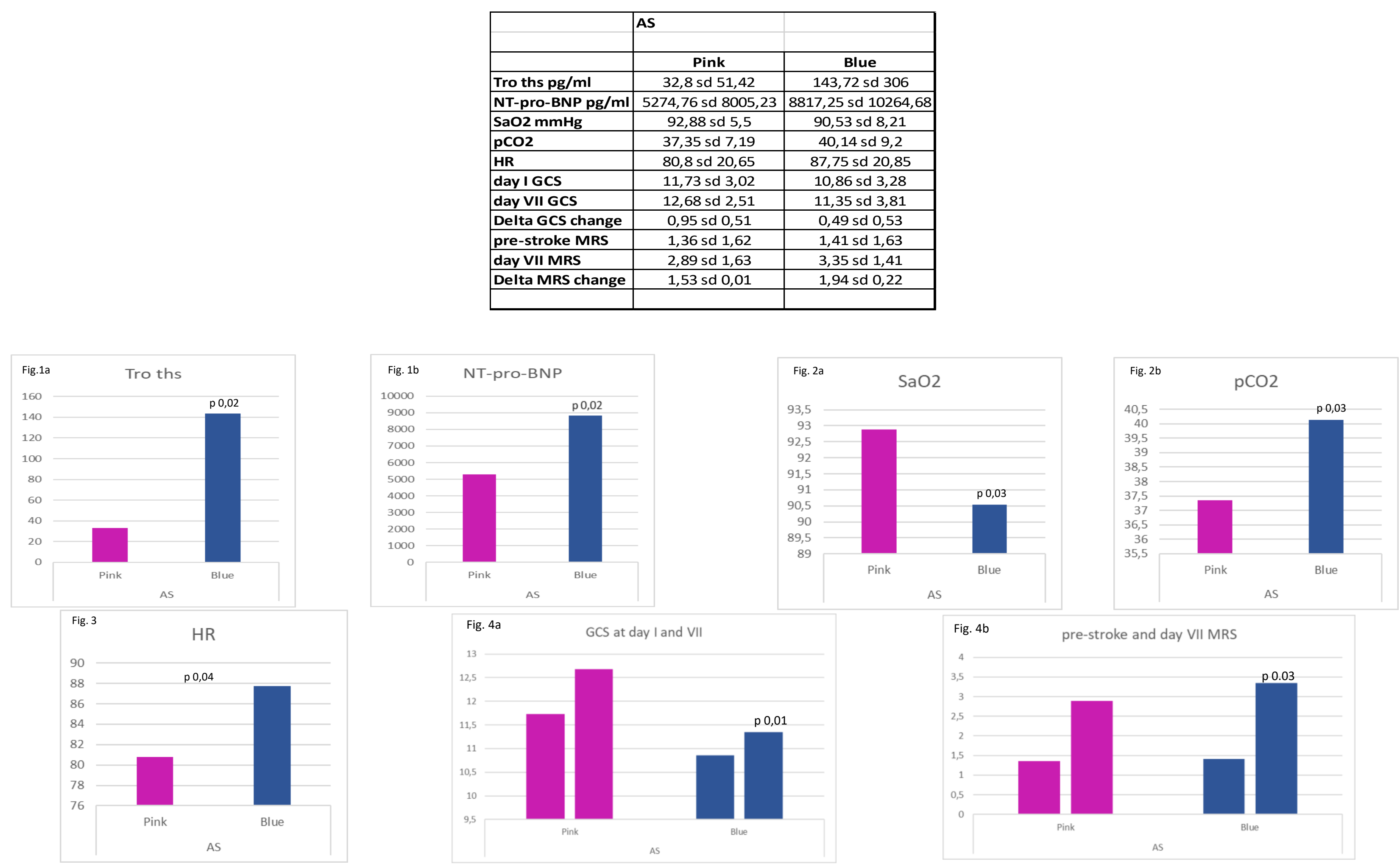